\begin{tabular}{|c|c|}
\hline Title & An Effective Robust Optimization Based on Genetic A Igorithm \\
\hline Author(s) & Maruyama, Takay uki; Igarashi, Hajime \\
\hline Citation & $\begin{array}{l}\text { IEEE Transactions on Magnetics, 44(6), 990-993 } \\
\text { https://doi.org/10.1109/1 MA G.2007.916696 }\end{array}$ \\
\hline Issue Date & $2008-06$ \\
\hline Doc URL & http:/hdl.handle.net $/ 2115 / 38721$ \\
\hline Rights & $\begin{array}{l}\text { @ 2008IEEE. Personal use of this material is permitted. However, permission to reprint/republish this material for } \\
\text { advertising or promotional purposes or for creating new collective works for resale or redistribution to servers or lists, } \\
\text { or to reuse any copyrighted component of this work in other works must be obtained from the IEEE. }\end{array}$ \\
\hline Type & article \\
\hline File Information & igarashi08-2.pdf \\
\hline
\end{tabular}

Instructions for use 


\title{
An Effective Robust Optimization Based on Genetic Algorithm
}

\author{
Takayuki Maruyama and Hajime Igarashi \\ Laboratory of Hybrid Systems, Graduate School of Information Science and Technology, Hokkaido University, Sapporo \\ 060-0814, Japan
}

\begin{abstract}
Although probabilistic optimization methods based on genetic algorithm (GA) provides accurate results, its performance is sometimes considerably sensitive to parameter changes. Moreover, the constraints are violated due to such parameter changes. A robust GA which performs random perturbation during optimization processes has been applied to some mathematical problems to show that it works as fast as the usual GAs. An adequate elite reservation technique for the robust GA is presented and applied to the robust GA for electromagnetic problems. Moreover, this method is shown to find solutions which are kept feasible against parameter changes.
\end{abstract}

Index Terms-Constraint condition, electromagnetic application, genetic algorithm (GA), robust optimization.

\section{INTRODUCTION}

I $\mathrm{N}$ the practical design of electromagnetic machines and devices, robust optimization with probabilistic methods such as genetic algorithm (GA) and immune algorithm plays a crucial role. However, robust optimization usually requires considerably great computational cost because exploration in the neighborhood of each individual must be performed for evaluation of its robustness. Robust optimization has, therefore, serious difficulties in applying for real optimization [1]-[3].

To overcome this difficulty, a novel method has been introduced, and proved to provide robust solutions with a computational cost which is comparable with that of nonrobust GAs [4]. This method has been shown to work well for one- or two- dimensional mathematical test problems.

It is important to test its validity for electromagnetic problems. To do so, an adequate elite conservation technique for the above method, which has not been mentioned anywhere, should be developed. Moreover, it is inconvenient in real situations that some manufacturing errors and external disturbances cause to violate constraint conditions. However, the robustness for constraints in this sense has not been discussed.

In this paper, we will introduce an effective elite selection algorithm for the novel method described above, which is called Robust GA (RGA) in this paper, to obtain robust optimal solutions under practical situations in computational electromagnetics. Moreover, RGA will be shown to suppress violation of constraints against parameter changes. Finally, we will apply this method to numerical problems which has constraint and TEAM workshop problem \#22 [5] to test its usefulness.

\section{METHODS}

While most parts of RGA processes are based on simple GAs, there is a difference in the evaluation process. Let us consider an optimization problem with single objective function $f(\boldsymbol{x})$. In GA optimization, $f(\boldsymbol{x})$ is evaluated for each individual. On the other hand, $f(\boldsymbol{x}+\boldsymbol{\delta})$ is evaluated instead in RGA, where $\boldsymbol{\delta}$ denotes noise vector with uniform or Gaussian distribution, which varies stochastically in the optimization process. When we consider $f(\boldsymbol{x}+\boldsymbol{\delta})$ instead of $f(\boldsymbol{x})$, points at the top of sharp peaks

Digital Object Identifier 10.1109/TMAG.2007.916696 would not have good performance. This property is theoretically shown in the following [4]. The mean of fitness in a GA is defined as

$$
\begin{aligned}
\overline{f(t)} & =\sum_{i=1}^{N} f\left(\boldsymbol{x}^{i}\right) / N \\
& =\int f(\boldsymbol{x}) p(\boldsymbol{x}, t) d \boldsymbol{x}
\end{aligned}
$$

where $p(\boldsymbol{x}, t)$ is continuous density function of individual distribution. On the other hand, the mean of fitness in RGA is represented as

$$
\begin{aligned}
\overline{f^{\prime}(t)} & =\sum_{i=1}^{N} f\left(\boldsymbol{x}^{i}+\boldsymbol{\delta}^{i}\right) / N \\
& =\int_{x} d \boldsymbol{x} \int_{\delta} f(\boldsymbol{x}+\boldsymbol{\delta}) p(\boldsymbol{x}, t) q^{\prime}(\boldsymbol{\delta}) d \boldsymbol{\delta} \\
& =\int_{x}\left[\int_{\delta} f(\boldsymbol{x}+\boldsymbol{\delta}) q^{\prime}(\boldsymbol{\delta}) d \boldsymbol{\delta}\right] p(\boldsymbol{x}, t) d \boldsymbol{x} \\
& =\int_{\delta} F(\boldsymbol{x}) p(\boldsymbol{x}, t) d \boldsymbol{x}
\end{aligned}
$$

where $\delta$ is noise vector and $q^{\prime}(\boldsymbol{\delta})$ is the distribution of $\delta$. Equations (1) and (2) show that $F(\boldsymbol{x})$ is an equivalent fitness function instead of $f(\boldsymbol{x})$ in RGA. The typical profiles of $f(\boldsymbol{x})$ and $F(\boldsymbol{x})$ are shown in Fig. 1. In (a) and (b), flat pulse functions with broad and relatively sharp profiles are assumed as $f(\boldsymbol{x})$, respectively. They correspond to robust and nonrobust solutions. The equivalent fitness $F(\boldsymbol{x})$ in (a) has better performance at the peak than that in (b) due to the board peak. Note here that $F(x)$ represents the expected value of $f(\boldsymbol{x}+\boldsymbol{\delta})$. In optimization processes, $f(\boldsymbol{x}+\boldsymbol{\delta})$ is evaluated because its distribution would approach $F(\boldsymbol{x})$ in an asymptotic way.

Although the elite selection method is often used to improve the convergence in GA, it is not used in the original RGA. Because the fitness of each individual is changed by the effect of noise, usual elite selection cannot be applied. In this paper, a new elite selection method is introduced as follows.

1) In generation $t$, search for the best individual and reserve it as an elite $\boldsymbol{x}_{t}$.

2) In generation $(t+1)$, search for an elite candidate $\hat{\mathbf{x}}_{t+1}$.

3) Compare $f\left(\boldsymbol{x}_{t}+\boldsymbol{\delta}\right)$ with $f\left(\hat{\boldsymbol{x}}_{t+1}\right)$. 


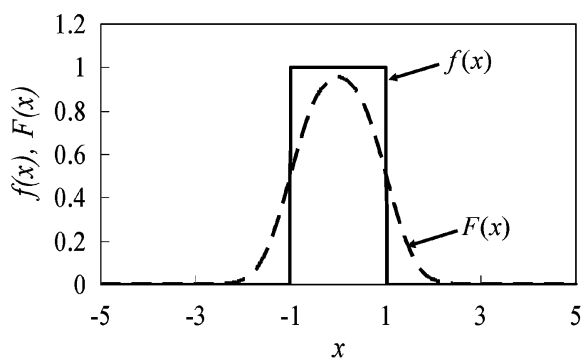

(a)

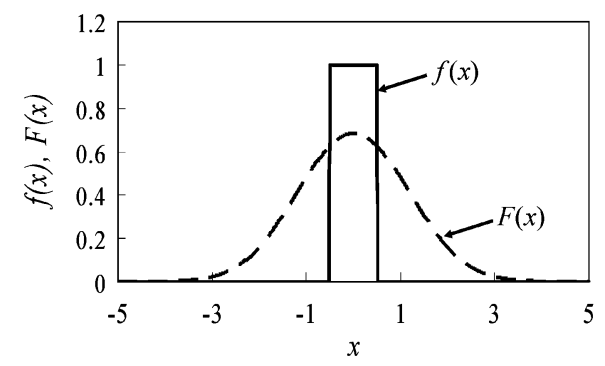

(b)

Fig. 1. Relation between $f(x)$ and $F(x)$. In RGA, $F(x)$ defined by (2) is used to evaluate individuals instead of $f(x) . F(x)$ takes lower value for sharp peaks. (a) Broad peak. (b) Sharp peak.

4) If $f\left(\hat{\boldsymbol{x}}_{t+1}\right)$ is better than $f(\boldsymbol{x} t+\boldsymbol{\delta}), \hat{\boldsymbol{x}}_{t+1}$ is reserved as a new elite. Otherwise, reserve $\boldsymbol{x t}$ as an elite.

5) Go to next generation and return to 2).

In process 3 ), the reserved elite $\boldsymbol{x}_{t}$ is evaluated with variation $\boldsymbol{\delta}$ to test whether $\boldsymbol{x}_{t}$ has enough robustness or not. The elite candidate $\hat{\boldsymbol{x}}_{t+1}$ is evaluated without noise because the elite must have good performance at least with its original parameters.

\section{RESULTS}

In this section, numerical results obtained by the present method are discussed. First of all, we report optimization of a simple rectangular function of a one-dimensional parameter. Then, we describe optimization of a two-dimensional mathematical function with a constraint. Finally, we report results for an optimization problem of superconducting magnets.

\section{A. One-Dimensional Rectangular Function}

In this problem, the objective function $f(x)$ is defined as

$$
f(x)= \begin{cases}2 & (1.0 \leq x \leq 1.4) \\ 1 & (3.0 \leq x \leq 5.0) \\ 0 & \text { (otherwise) }\end{cases}
$$

According to (3), $f(x)$ has two peaks; one is a sharp, nonrobust peak where $f(x)=2$ and the other is a broad robust peak where $f(x)=1$. GA parameters are shown in Table I.

The noise rate is set to $10.0 \%$. The result obtained by conventional GA is shown in Fig. 2. The distribution of individuals in the last generation is plotted in Fig. 2. Cleary, all individuals converge at the nonrobust peak.

The result obtained by the present method is shown in Fig. 3 . Most of individuals are converged to the broad peak or near the
TABLE I

GA PARAmeters (MATHEMATICAL PRoBlem)

\begin{tabular}{lr}
\hline Population size & 20 \\
Trials & 1000 \\
Probability of crossover & 1.0 \\
Probability of mutation & 0.05 \\
Generation gap & 1.0 \\
\hline
\end{tabular}

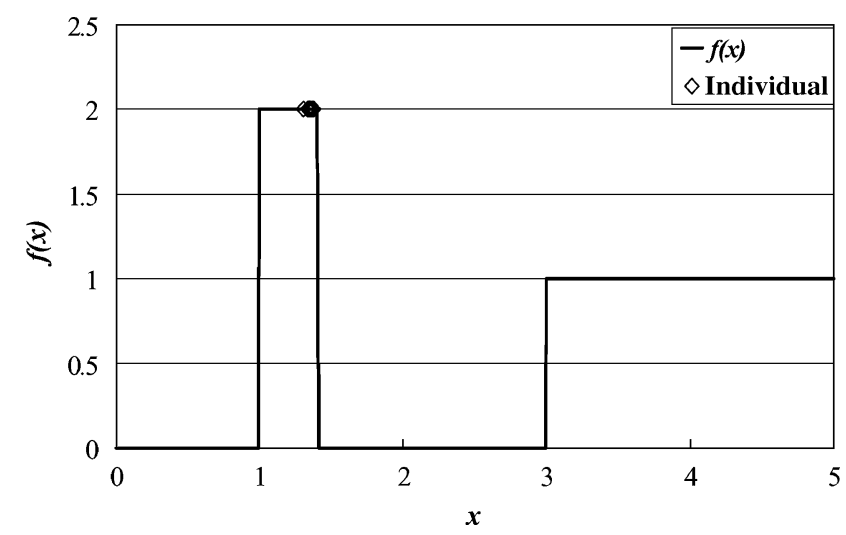

Fig. 2. Optimal solutions obtained by GA. Individuals cluster on the edge of the sharp peak.

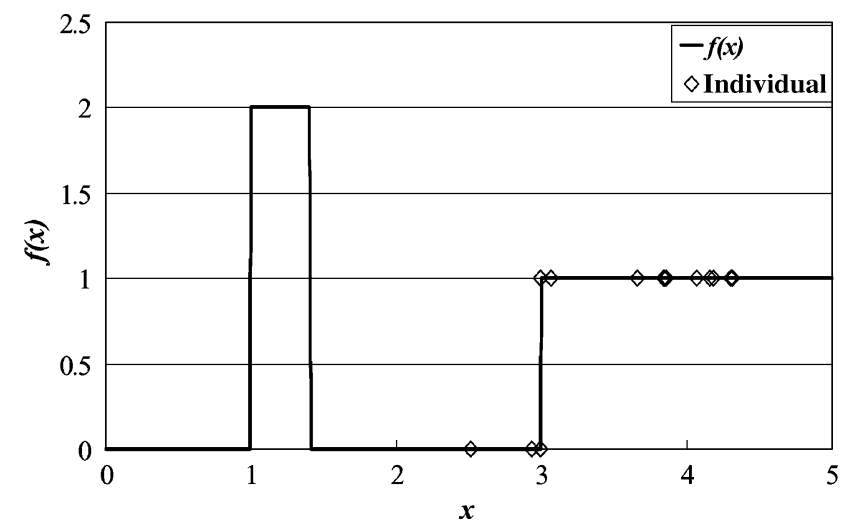

Fig. 3. Optimal solutions obtained by the present method. Individuals cluster on the center of the broad robust peak.

broad peak. It is concluded that the present method can converge to robust solutions.

\section{B. Mathematical Function With Constraint}

Here, we consider an optimization with a constraint using the present method. The objective function is defined as

$$
f\left(x_{1}, x_{2}\right)=1.0+\frac{\cos \left(\pi x_{1}\right)+\cos \left(\pi x_{2}\right)}{2} .
$$

The domains of $x_{1}$ and $x_{2}$ range from 0.0 to 1.0 , which must meet the following constraint:

$$
x_{1}^{2}+x_{2}^{2} \geq \varepsilon^{2}
$$

where $\varepsilon$ is set to 0.1 . If an individual violates this constraint, its evaluated value is decreased to zero. In this problem, ob- 


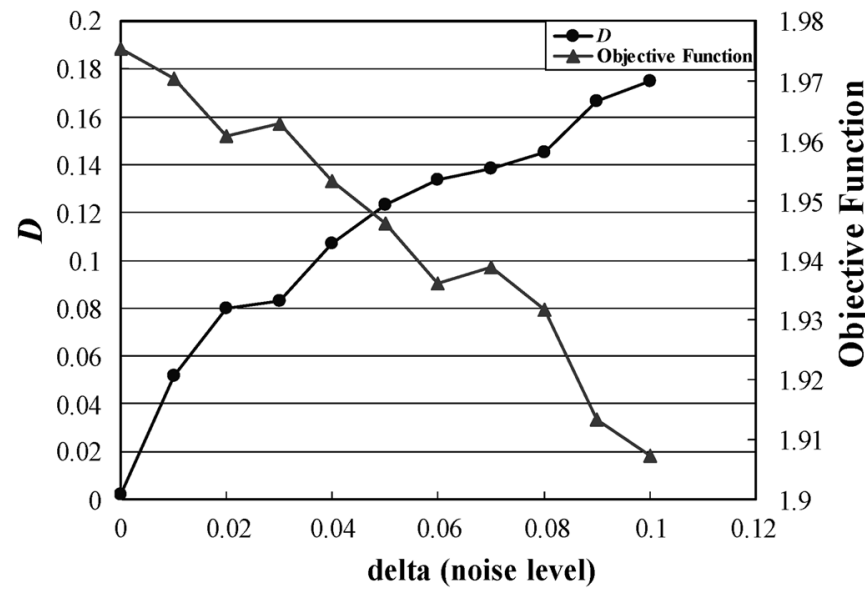

Fig. 4. Mean values of $D$ and resultant objective function. There is a tradeoff between robustness for constraint and the performance.

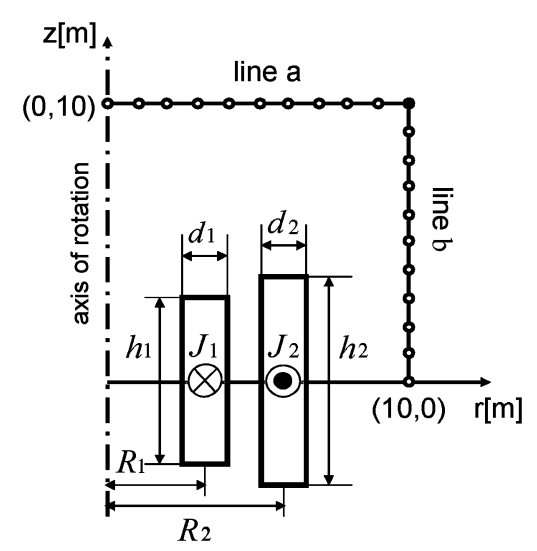

Fig. 5. SMES model.

tained optimal solutions are evaluated their robustness for the constraint by using following quantity:

$$
D=\sqrt{\varepsilon^{2}-\left(x_{1}^{2}+x_{2}^{2}\right)}
$$

where $D$ means the distance between the solution and the border of constraint denoted by (5). Thus, the solution which has small $D$ easily violates the constraint condition because of small parameter changes or disturbances.

The GA parameters are again set to the values listed in Table I. The noise ranges from 0.00 to 0.10 . Optimization is performed with different noises. To obtain mean values of $D$ and objective function, ten trials are carried out. The result is shown in Fig. 4, where the mean of $f\left(x_{1}, x_{2}\right)$ and $D$ is plotted. The result shows that large noise keeps solutions far away from a constraint border, but it makes objective function worse. Thus, there is a tradeoff between objective function and robustness for constraint. In real situations, the noise level would be determined from degree of uncertainty in the parameters or production tolerances.
TABLE II

GA PARAMETERS (SMES PROBLEM)

\begin{tabular}{lr}
\hline Population size & 100 \\
Trials & 4000 \\
Probability of crossover & 1.0 \\
Probability of mutation & 0.05 \\
Generation gap & 1.0 \\
\hline
\end{tabular}

TABLE III

PARAMETERS OF OPTIMUM SOLUTION

\begin{tabular}{lccccc}
\hline & $\begin{array}{c}R_{2} \\
{[\mathrm{~m}]}\end{array}$ & $\begin{array}{c}h_{2} \\
{[\mathrm{~m}]}\end{array}$ & $\begin{array}{c}d_{2} \\
{[\mathrm{~m}]}\end{array}$ & $\begin{array}{c}J_{1} \\
{\left[\mathrm{~A} / \mathrm{mm}^{2}\right]}\end{array}$ & $\begin{array}{c}J_{2} \\
{\left[\mathrm{~A} / \mathrm{mm}^{2}\right]}\end{array}$ \\
\hline GA & 3.19 & 4.00 & 0.10 & 23.90 & -10.34 \\
$\operatorname{RGA}(0.5 \%)$ & 3.20 & 3.99 & 0.10 & 23.87 & -10.27 \\
$\operatorname{RGA}(1.0 \%)$ & 3.12 & 3.97 & 0.10 & 24.02 & -10.66 \\
RGA $(1.5 \%)$ & 3.15 & 3.64 & 0.11 & 24.01 & -10.89 \\
\hline
\end{tabular}

GA is the Conventional GA Without Robustness Treatment, same in Tables IV and V.

\section{Optimization of Electromagnetic Systems}

The model of superconducting magnetic energy storage (SMES) is shown in Fig. 5 [5]. This problem has eight parameters. The parameters $\left(R_{1}, h_{1}, d_{1}\right)$ and $\left(R_{2}, h_{2}, d_{2}\right)$ in Fig. 5 denote the sizes of two superconducting coils. Moreover, $J_{1}$ and $J_{2}$ denote current densities of each coils.

In the SMES optimization problem, it is necessary that the storage energy is fulfilled the prescribed value and the total stray magnetic field on line $a$ and line $b$ is minimized. Thus, the following objective function is minimized:

$$
O F=\frac{B_{\text {stray }}^{2}}{B_{\text {norm }}^{2}}+\frac{\mid \text { Energy }-E_{\text {ref }} \mid}{E_{\text {ref }}}
$$

where $B_{\text {norm }}$ and $E_{\text {ref }}$ is set to $3[m T]$ and $180[M J]$, respectively. Moreover, the optimal solution is required to fulfill following constraint:

$$
|\boldsymbol{J}| \leq(-6.4|B|+54.0)\left[\mathrm{A} / \mathrm{mm}^{2}\right] .
$$

If an individual violates this constraint condition, it means that superconducting state cannot be sustained. The phenomenon to transit from superconducting state to normal state is called quench. In the SMES problem, it is especially important to keep the superconducting constraint given by (8).

GA parameters in this problem are shown in Table II. Here, $R_{1}, h_{1}$, and $d_{1}$ are set to $2.00,1.60$, and $0.27[\mathrm{~m}]$, respectively, and other five parameters are optimized. The uniform noise is added to $R_{2}, h_{2}$, and $d_{2}$. The noise levels are increased from $0.5 \%$ to $1.5 \%$. The obtained solutions are evaluated their robustness a posteriori by repeatedly calculating objective function with $0.5 \%$ noise. Moreover, the quench rate, which is a quench probability evaluated with $0.5 \%$ noise, is evaluated. The quench rate is calculated by the number of times the obtained solution violates the constraint in the ex post facto evaluation. High quench rate means high possibility for violation of superconducting state.

The optimization results are shown in Tables III and IV. Table III shows parameters of obtained solutions. Table IV shows the result of ex post facto evaluation. $O F$ in Table IV represents the value of objective function without noise. Worst 
TABLE IV

RESULT OF EX POST FACTO EVALUATION

\begin{tabular}{ccccc}
\hline & GA & $\begin{array}{c}\text { RGA } \\
(0.5 \%)\end{array}$ & $\begin{array}{c}\text { RGA } \\
(1.0 \%)\end{array}$ & $\begin{array}{c}\text { RGA } \\
(1.5 \%)\end{array}$ \\
\hline OF & $1.48 \mathrm{E}-02$ & $1.71 \mathrm{E}-02$ & $2.10 \mathrm{E}-02$ & $2.99 \mathrm{E}-02$ \\
Worst OF & $3.81 \mathrm{E}-02$ & $3.84 \mathrm{E}-02$ & $3.79 \mathrm{E}-02$ & $4.66 \mathrm{E}-02$ \\
$\Delta O F$ & $2.33 \mathrm{E}-02$ & $2.13 \mathrm{E}-02$ & $1.69 \mathrm{E}-02$ & $1.67 \mathrm{E}-02$ \\
\hline
\end{tabular}

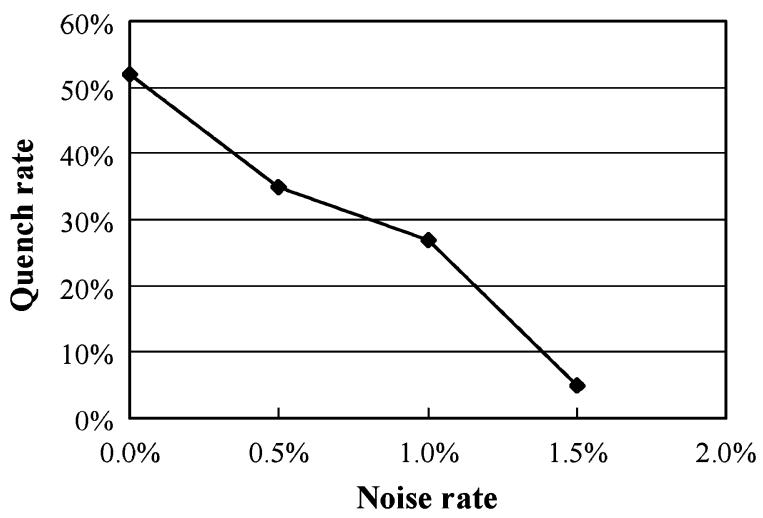

Fig. 6. Dependence of quench rate on noise rate. Solutions optimized with large noise have higher feasibility.

$O F$ represents the worst value of objective function in the evaluation. And $\triangle O F$ is defined by Worst $O F-O F$. The noise parameter improves somewhat $\triangle O F$ in this case. However, it is possible that too large a noise worsens the performance.

The relation between noise level and quench rate is shown in Fig. 6. The solution obtained by original GA has a very high quench rate. Such a solution has difficulty to keep a superconducting state because it requires very small manufacturing errors and very strict control of currents. The solutions obtained by the present method has low quench rate when with enough noise level.

Table $\mathrm{V}$ shows required CPU time. It shows that the present method can obtain solutions, which are robust with respect to performance and feasibility, with almost the same computational cost as that of GA. For reference, the conventional robust
TABLE V

REQUIRED CPU TIME

\begin{tabular}{lcccc}
\hline & GA & $\begin{array}{c}\text { RGA } \\
(0.5 \%)\end{array}$ & $\begin{array}{c}\text { RGA } \\
(1.0 \%)\end{array}$ & $\begin{array}{c}\text { RGA } \\
(1.5 \%)\end{array}$ \\
\hline CPU time [min] & 259.1 & 248.8 & 259.2 & 252.2 \\
\hline
\end{tabular}

CPU: Intel Xeon3.06 GHz (FSB533)

optimization method which repeats sensitivity analysis in its process [3] requires about $2150 \mathrm{~min}$ to solve the same problem in our experience.

\section{CONCluSION}

In this paper, an effective robust optimization method with a new elite selection method is introduced. The present method can obtain robust solutions without extra computational cost. Moreover, the present method is shown to converge to a solution which is far from the border of constraints. Thus, the solutions obtained by the present method have high feasibility. However, they have lower performance than nonrobust solutions, although this could not be avoidable. That is, the robustness and/or feasibility have a tradeoff relation with performance.

\section{REFERENCES}

[1] X. Du and W. Chen, "Towards a better understanding of modeling feasibility robustness in engineering design," J. Mech. Des., vol. 122, pp. 385-394, 2000.

[2] K.-H. Lee and G.-J. Park, "Robust optimization considering tolerances of design variables," Comput. Structures, vol. 79, pp. 77-86, 2001.

[3] G. Steiner, A. Weber, and C. Magele, "Managing uncertainties in electromagnetic design problems with robust optimization," IEEE Trans. Magn., vol. 40, no. 2, pp. 1094-1099, Mar. 2004.

[4] S. Tsutsui and A. Ghosh, "Genetic algorithms with robust solution searching scheme," IEEE Trans. Evol. Comput., vol. 1, no. 3, pp. 201-208, Sep. 1997.

[5] "TEAM Workshop Problem \#22: SMES Optimization Benchmark," TEAM Workshop [Online]. Available: http://www.igte.tugraz.at/archive/te-am_new/description.php

Manuscript received June 24, 2007. Corresponding author: T. Maruyama (e-mail: maruyama@em-si.eng.hokudai.ac.jp). 